\title{
Provision of rapid HIV tests within a health service and frequency of HIV testing among men who have sex with men: randomised controlled trial
}

\author{
(a) (1) $\Theta$ OPEN ACCESS
}

\author{
Tim R H Read research scholar ${ }^{12}$, Jane S Hocking associate professor ${ }^{3}$, Catriona S Bradshaw \\ associate professor ${ }^{124}$, Andrea Morrow research nurse ${ }^{1}$, Andrew E Grulich professor ${ }^{5}$, Christopher \\ $\mathrm{K}$ Fairley professor ${ }^{12}$, Marcus Y Chen associate professor ${ }^{12}$
}

\begin{abstract}
${ }^{1}$ Melbourne Sexual Health Centre, Alfred Health, Carlton, VIC 3053, Australia; ${ }^{2}$ Melbourne School of Population and Global Health, University of Melbourne, Melbourne, VIC 3010, Australia; ${ }^{3}$ Centre for Women's Health, Gender and Society, University of Melbourne, Melbourne, VIC 3010 , Australia; ${ }^{4}$ Department of Epidemiology and Preventive Medicine, Monash University, Melbourne, VIC 3004 , Australia; ${ }^{5}$ Kirby Institute, University of New South Wales, Kensington, NSW 2052, Australia
\end{abstract}

\begin{abstract}
Objective To determine if the provision of rapid HIV testing to men who have sex with men attending a health service would increase their frequency of HIV testing over time.

Design Non-blinded randomised controlled trial.

Setting Public sexual health service in Australia.

Participants Men who reported having a male sexual partner within the previous year and an HIV test within the previous two years. Of 400 men entered, 370 (92.5\%) completed the study.

Interventions Men attending the service between September 2010 and March 2011 were randomised 1:1 to either ongoing access to rapid HIV testing obtained with finger prick or to conventional HIV serology with venepuncture, over 18 months.

Main outcome measure The incidence of all HIV testing after enrolment, including testing outside the study clinic, analysed by intention to treat.

Results Of 200 men randomised to the rapid testing arm, 196 were followed for 288 person years. Of 200 men randomised to the conventional testing arm, 194 were followed for 278 person years. Median time since the last HIV test was six months for both arms. Men in the rapid test arm had 469 tests (mean 1.63 tests a year), and men in the conventional test arm had 396 tests (mean 1.42 tests a year); incidence rate ratio $1.15,95 \%$ confidence interval 0.96 to $1.38 ; P=0.12$. In a post hoc analysis, rates of initial HIV testing during follow-up were 1.32 and 1.01 tests a year, respectively $(1.32,1.05$ to $1.65 ; \mathrm{P}=0.02)$.
\end{abstract}

Conclusions Provision of access to rapid HIV testing in a health service did not result in a sustained increase over time in HIV testing by men who have sex with men; however, the rate of initial HIV testing did increase by a third. Further research is required to determine how to achieve sustained increases in the frequency of HIV testing by populations at risk.

Trial registration ACTR No 12610000430033.

\section{Introduction}

In many countries the prevalence and incidence of HIV remains high among men who have sex with men, despite prevention campaigns. ${ }^{1-4}$ Increased HIV testing in these men could help to stem HIV transmission because awareness of positive HIV status could reduce sexual risk behaviour ${ }^{5}{ }^{6}$ and because timely diagnosis provides an opportunity for earlier antiretroviral therapy, which would maximise the impact of treatment as prevention. ${ }^{7}$ The potential impact of HIV testing on transmission in a population depends in part on the proportion of infected individuals who are unaware of their HIV status. Mathematical models suggest that over $50 \%$ of HIV transmissions in the United States were from undiagnosed individuals ${ }^{8}$ and that more frequent HIV testing would reduce transmissions. ${ }^{9}{ }^{10}$

Seroprevalence surveys and surveillance indicate that $12-30 \%$ of HIV positive men who have sex with men in Australia, Canada, and New Zealand remain undiagnosed, rising to $44 \%$ in the US and $86 \%$ in one survey from China. ${ }^{11-17}$

Guidelines recommend that men who have sex with men should have at least annual HIV testing, with more frequent testing in men at higher risk. ${ }^{18-20}$ The public health benefits resulting from early diagnosis, treatment, and behaviour change are likely to be greater with more frequent testing because HIV is most infectious around the time of seroconversion, when the viral load is highest. ${ }^{21}{ }^{22}$ Data from high and middle income countries indicate that rates of HIV testing among men who have sex with men often fall short of the levels recommended. ${ }^{1823-25}$ 
Several impediments to HIV testing of men who have sex with men have been identified. These include fear of a positive result, anxiety while waiting for a result, difficulty in accessing services for testing, and the need to return to obtain results. ${ }^{26}$ Not all those infected with HIV who attend a clinic will be tested and diagnosed. ${ }^{28}$ Point of care tests for HIV, commonly referred to as rapid HIV tests, have the potential to improve the uptake of testing because of the immediacy of test results. When surveyed, most men who have sex with men in Melbourne anticipated they would test more frequently if oral fluid rapid HIV tests were available. In 2012 the US Food and Drug Administration approved the sale of self administered oral fluid tests in an effort to increase testing and reduce the pool of people with undiagnosed HIV. ${ }^{29} 30$

Several studies have shown that rapid tests increase uptake of HIV testing in various settings, though these have been cross sectional studies comparing the uptake of a rapid or conventional HIV test on a single occasion without examining the frequency of testing after the first test. ${ }^{31-35}$ To date there is no empirical evidence that the provision of rapid tests leads to a sustained increase in the frequency of HIV testing over time in any population. We undertook a randomised controlled trial to determine whether the provision of rapid tests for HIV within a health service would increase the frequency of HIV testing among men who have sex with men.

\section{Methods}

\section{Trial design}

In this non-blinded trial, the men were randomised in a 1:1 ratio to either ongoing access at the health service to rapid tests for HIV (intervention) or to conventional HIV testing (control) at the same health service over an 18 month period. Recruitment took place from September 2010 to March 2011 with the last participant completing 18 months of follow-up in September 2012.

\section{Participants}

This study took place at the Melbourne Sexual Health Centre, the major public clinic for sexually transmitted diseases in Victoria, Australia. Eligible participants were men aged $\geq 18$ attending for clinical care who reported having sex with a man within the previous year and who had had a negative HIV test result within the previous two years. To increase the likelihood that men would retest within the study period we recruited only men who had been tested for HIV within the previous two years. Men seeking post-exposure prophylaxis for HIV were excluded from the study as were those planning to live outside Victoria for more than six months.

\section{Interventions}

Men randomised to the intervention arm were tested at enrolment with the Determine HIV-1/2 Antigen/Antibody Combo test (Alere, Japan) in whole blood obtained from finger pricks. These men were informed that they could attend the clinic at any time over the subsequent 18 months to be tested for HIV with a rapid test. Men randomised to the control arm were offered the clinic's standard HIV test: venepuncture with serum forwarded to the Victorian Infectious Diseases Reference Laboratory for testing by third generation enzyme immunoassay (Murex, Dartford, UK). Men in the control arm were required to return to the clinic one week after the test so they could be given the HIV result in person. Men tested with rapid tests received their result 20 minutes after the finger prick.
The clinic protocol governing testing for HIV and sexually transmitted infections in men who have sex with men followed Australian guidelines-namely, annual screening for HIV, syphilis, pharyngeal and rectal gonorrhoea, and urethral and rectal chlamydia, with three to six monthly screening of men at higher risk. ${ }^{19}$ Screening for syphilis was undertaken by venepuncture and serology. Men in both arms were required to undergo venepuncture if they agreed to syphilis testing.

Reactive antibody results on rapid testing were confirmed by the Murex third generation enzyme immunoassay-the Genscreen antigen-antibody HIV test (Bio-Rad, France) - and by western blot. Indeterminate results were resolved by testing of a further serum sample obtained at least two weeks later. Reactive rapid antigen results were confirmed by P24 antigen EIA (Bio-Rad, France).

Men in both arms of the study were managed in the same way according to the standard clinical pathways and management protocols when they attended for subsequent visits, apart from the type of HIV test that they could have. This included access to the clinic without a previous appointment and brief assessment by a triage nurse followed by a consultation with a nurse or doctor who then discussed and offered testing for sexually transmitted infections and HIV. Men having an HIV test were referred to a nurse who performed the finger prick test for men in the intervention arm or venepuncture for men in the control arm. Men in the intervention arm were free to have conventional HIV testing if they wanted.

Men in both arms of the study were sent text messages at months three, nine, and 15 of the study recommending regular HIV testing and offering either an "HIV test" or a "rapid HIV test" at the study clinic, according to their allocated arm. They were also referred via the text message to a website that was specific for their allocated arm to remind them of the type of testing available to them and were given a dedicated study phone number for inquiries. The electronic clinic medical records were marked to indicate to clinicians that patients were participants in the trial and noted the arm to which they had been allocated. Men in both arms received email messages at months six, 12, and 18 containing a link to an online study questionnaire. A $\$ A 20(£ 12, € 14, \$ 18)$ voucher was offered to participants who completed all questionnaires.

\section{Outcomes}

Our primary outcome was the frequency (incidence rate) of HIV testing over 18 months, expressed as number of tests per person year, excluding tests performed at enrolment. HIV testing included HIV testing performed at the study clinic, whether rapid or conventional, as well as HIV testing performed by healthcare providers at other clinics. No rapid tests for HIV were approved for clinical use in Australia during the study period and therefore all testing at other sites would have been through conventional serology. HIV testing and results were recorded in the electronic medical records system.

Men were asked via the online questionnaires if they had been tested for HIV elsewhere and, if so, when and where this had taken place. If questionnaires were not completed this information was obtained through text messages sent to individual men. Researchers then contacted external clinics to verify that HIV testing had occurred and the date of testing. The online questionnaires included questions about attitudes to HIV testing and sexual behaviour. Participants were also asked how they felt about their HIV test experience. The secondary outcome was the frequency (incidence rate) of testing for syphilis, gonorrhoea, and chlamydia to determine if rapid HIV testing 
would result in a fall in testing for other sexually transmitted infections.

\section{Sample size}

Before this study the mean interval between HIV tests among men who have sex with men who were attending the clinic and who had previously tested within the past two years was 12 months (SD 5 months). A $10 \%$ increase in the proportion of men tested annually would have reduced the interval between tests by six weeks. Because modelling of the Australian HIV epidemic suggested that a $10 \%$ increase would avert 13 new HIV infections each year, we powered the study to detect a minimum difference in interval between tests of six weeks. ${ }^{10}$ If we assume a mean interval between HIV tests of 12 months, a study with 174 people in each arm would have $80 \%$ power to detect a 1.5 month reduction in this interval at a significance of 0.05 .

\section{Randomisation and blinding}

Participants were randomised with a randomised block design with two computer generated random sequences per block (prepared by JSH) to ensure there would be equal numbers allocated to each study arm. A research assistant not associated with the trial sealed study allocations in numbered opaque envelopes that were opened in sequence at the time each man was enrolled. Research personnel who enrolled participants and assigned interventions (AM and TRHR) were unaware of the allocation until the envelope was opened in front of the patient. The study design required participants and clinic staff to be aware of the allocated type of HIV test.

\section{Statistical methods}

We calculated the incidence of HIV testing with intention to treat analysis after excluding individuals with positive HIV test results at enrolment. Men who did not have a subsequent test and those who were lost to follow-up were censored at their last contact when questionnaires were sent at six months, 12 months, or at study end at 18 months. Men who developed incident HIV infection during follow-up were censored at the time of their new positive test result. Confirmatory HIV testing was excluded from the analysis. We used Poisson regression methods to estimate the ratio of incidence rates of HIV testing in the rapid test arm compared with the control arm and calculated robust standard errors to account for repeated measures from individuals. Proportions and 95\% confidence intervals were calculated with binomial exact methods. Differences between proportions were investigated with the $\chi^{2}$ statistics. Analyses were undertaken with Stata version 11.

\section{Results}

From September 2010 to March 2011, clinicians referred 445 men to the study. Of these men, 26 declined to participate (fig $1 \Downarrow$ ). Nineteen men were found to be ineligible, mainly because they were not planning to reside within Victoria during the study period. Of the 400 men who were recruited and randomised, 200 were assigned to HIV rapid testing and 200 to conventional testing. Among the 200 men randomised to rapid testing, five had a diagnosis of with HIV (two at baseline), six were lost to follow-up, and 189 (94.5\%) completed 18 months of follow-up. Among the 200 men randomised to conventional testing five had a diagnosis of HIV (three at baseline), 14 were lost to follow up, and $181(90.5 \%)$ completed 18 months of follow-up (fig $1) \Downarrow$. The 10 men with a diagnosis of HIV were censored at the date of their positive result. Five were positive at enrolment (overall baseline prevalence $1.25 \%$, (95\% confidence interval $0.4 \%$ to $2.9 \%$ ) and five were incident cases (overall incidence $0.9 \%$ a year, $0.3 \%$ to $2.1 \%$ ). Table 1 compares the characteristics of men in the intervention and control arms $\Downarrow$.

Men assigned to the rapid test arm had 469 tests during 288 person years of follow-up while men in the conventional test arm had 396 tests during 278 person years of follow-up. After enrolment, the incidence of HIV testing in the rapid and conventional test arms over 18 months was 1.63 and 1.42 tests a year, respectively, representing an incidence rate ratio of 1.15 ( $95 \%$ confidence interval 0.96 to $1.38 ; \mathrm{P}=0.12$ ) (table $2 \Downarrow$ ). When we included only the first HIV test after enrolment in a post hoc analysis, the rate of HIV testing in the rapid test arm was higher than in the conventional test arms. The rate was 1.32 tests a year (161 tests/122 person years) in the rapid test arm and 1.01 tests a year (141 tests/140 person years) in the conventional test arm, representing an incidence rate ratio of 1.32 (10.5 to $1.65 ; \mathrm{P}=0.02$; table 2, fig $2 \Downarrow$ ). When we excluded the first HIV test after enrolment, the rate of subsequent HIV testing was the same in both arms, at 1.86 tests a year in the rapid arm and 1.83 tests a year in the conventional arm (1.01, 0.86 to $1.20 ; \mathrm{P}=0.90)$.

The rate of testing for syphilis, chlamydia, and gonorrhoea at the study clinic did not differ significantly between study arms. The rates of syphilis testing in the intervention and control arms were 1.42 and 1.32 tests a year, respectively (incidence rate ratio $1.13,95 \%$ confidence interval 0.95 to $1.35 ; \mathrm{P}=0.18$ ), while the rates of testing for chlamydia and gonorrhoea were 1.56 and 1.42 a year, respectively $(1.11,0.90$ to $1.36 ; \mathrm{P}=0.33)$.

Unconfirmed reactive tests, representing false positive results, were more common with rapid tests than with conventional serology $(9 / 596,1.5 \%$ (95\% confidence interval $0.6 \%$ to $2.8 \%$ ) $v 1 / 534,0.2 \%$ ( $0 \%$ to $1.0 \%) ; \mathrm{P}=0.02$ ). Of 417 tests performed in the study clinic after enrolment in the rapid test arm, 396 $(95 \%)$ were rapid tests and $21(5 \%)$ were conventional tests. Reasons for the performance of conventional tests among participants in the rapid test arm included declined rapid testing after a previous false positive rapid test result $(n=5)$, declined rapid testing without providing a reason $(n=6)$, and clinic staff were unaware of the study $(n=5)$.

At the baseline visit, men in the intervention arm were asked about their preference for HIV testing after they had experienced the finger prick test. Most men (167/190, 88\%, 95\% confidence interval $82 \%$ to $92 \%$ ) said they preferred rapid tests over conventional HIV testing. The final study questionnaire was completed by $270 / 390(69 \%)$ of the men who remained HIV negative throughout the study: $142 / 195(73 \%)$ in the rapid test arm and 128/195 (66\%) in the conventional serology arm $(\mathrm{P}=0.10)$. Compared with men randomised to rapid tests, men with access only to conventional serology were more likely to feel that the wait for the test result was too long (75/128 (59\%) $v 13 / 142(9 \%), \mathrm{P}<0.001)$, to report anxiety because of the wait $(81 / 128(63 \%) v 63 / 142(44 \%), \mathrm{P}=0.002)$, and to report delaying their next test because of anxiety over the wait (30/127 (24\%) $v 19 / 142$ (13\%), $\mathrm{P}=0.03)$. More men randomised to rapid tests reported that obtaining their HIV test result was convenient $(105 / 141(74 \%)$ v 52/128 (41\%), $\mathrm{P}<0.001)$.

\section{Discussion}

In this study, provision of rapid HIV testing to men who have sex with men attending a health service did not result in a sustained increase in their frequency of testing over time when compared with conventional HIV testing. In a post hoc analysis, however, the rate of initial HIV testing did increase by a third. To our knowledge this is the first randomised controlled trial 
to measure the effect of an intervention on the frequency of HIV testing in a cohort of individuals followed over time. Our findings have implications for the development and evaluation of new approaches, including the use of rapid testing, aimed at achieving sustainable increases in HIV testing within populations at risk.

Several aspects of this study need to be considered in the interpretation of the results and their relevance to other settings. Firstly, rapid tests had to be performed in the clinic after a clinical consultation, which kept participants in the clinic longer than would have been necessary for rapid testing alone. The time required for this process could have deterred more frequent testing in the rapid test arm. Secondly, we enrolled men who had been tested for HIV within the past two years and were therefore predisposed to testing. Rapid testing might have a greater effect on the testing frequency of men who have never had HIV tests or who test less frequently than the men in our study. Furthermore, men undergoing conventional HIV testing were required to return to the clinic for their results. This could have discouraged more frequent testing in the conventional HIV testing arm and increased the apparent effect of the intervention. The intervention might have had less effect in a health service that did not require a return visit for test results. Finally, the study was powered to detect a six week reduction in the mean interval between HIV tests, which we determined would probably only deliver a marginal public health benefit for men who have sex with men in Australia. But a smaller change in the interval between tests could be important in populations with a higher incidence of HIV, as seen in some epidemics in resource poor countries.

\section{Results in context}

Other studies have compared the acceptance of rapid and conventional HIV tests but differ from ours as they have measured uptake on only one occasion, without examining whether uptake is sustained over time. In these studies, which include randomised trials in diverse settings, the uptake of rapid testing was consistently higher. ${ }^{31} 323435$ In addition to greater uptake of testing, a higher proportion of those undergoing rapid HIV testing received their test results compared with those who had conventional tests. ${ }^{31} 33$ In one randomised study, acceptance of HIV testing did not differ between individuals offered oral fluid and finger prick rapid tests, ${ }^{32}$ suggesting that use of rapid finger prick testing rather than oral fluid testing in this study was unlikely to have reduced the effect of the intervention. Randomised trials of other interventions-such as educational videos or mobile testing services-have examined the proportion of a population having one or more tests after an intervention but have not followed the testing frequency of individuals over an extended period. ${ }^{36}$ Interventions aimed at increasing HIV testing need to achieve sustained increases if they are to confer meaningful individual and public health benefits. Evaluations need to assess the durability of such interventions over time.

Men who had access to the rapid HIV tests in our study preferred them over conventional tests and expressed less concern about some of the recognised barriers to HIV testing, such as the wait for the test result and the inconvenience of testing. This probably explains the observed higher rate of return for initial HIV testing after enrolment. But simply offering rapid testing within a health service was not enough to bring about a sustained rise in testing. Policymakers and clinicians should be cautious about assuming this will occur without considering the setting. Rapid tests might be more effective in locations that are more easily accessible to target populations, such as shop front testing sites or even at home. One advantage of rapid HIV tests is that they improve the likelihood that HIV positive individuals receive their results. Though this is important in settings where effective provision of results is difficult, it rarely occurred in our setting and was therefore not a study outcome. ${ }^{38}$

Men who have sex with men remain one of the major risk groups for HIV globally and strategies to reduce the number of undiagnosed HIV infections are urgently required. Whether provision of rapid testing in countries with good access to laboratory HIV testing will boost the frequency of HIV testing is not known. Studies of novel, cost effective strategies to reduce barriers to HIV testing and enhance testing among men who have sex with men and other populations at risk are needed. It is vital that such studies are conducted with a sufficiently long follow-up period to determine the durability of their effect.

We thank all the men who participated in this study; Joe Vincini, National Serological Reference Laboratory; Jody Mitchell and Deborah Phillips, Alere (Australia and New Zealand); David Wilson, Kirby Institute; Alan Breschkin, Victorian Infectious Diseases Reference Laboratory; Simon Powell Centre Clinic; Lisa Millett, Northside Clinic; Andrea Edwards, Prahran Market Clinic; and Lenka Vodstrcil, Huachun Zou, Melanie Bissessor, David Lee, Anthony Snow, Clare Bellhouse, and all clinical staff at Melbourne Sexual Health Centre, Alfred Health.

Contributors: TRHR, CKF, and MYC designed and were principal investigators of the study, with assistance from CSB. AM performed most of the rapid tests, confirmed tests performed elsewhere, and collated data for this study. JSH and TRHR analysed the data, with advice from AEG. All authors contributed to the final report. CKF and MYC are joint last authors. TRHR and MYC are guarantors.

Funding: The study was funded by National Health and Medical Research Council of Australia program grant No 568971. TRHR is the recipient of an Australian postgraduate award. Only the Melbourne Sexual Health Centre had access to the study data, and the authors had sole responsibility of decision for publication.

Competing interests: All authors have completed the ICMJE uniform disclosure form at www.icmje.org/coi_disclosure.pdf (available on request from the corresponding author) and declare that Alere (Australia and New Zealand) provided free rapid test kits and provided advice on quality control but had no input into the design, analysis or reporting of this study.

Ethical approval: This study was approved by the Alfred Hospital research ethics committee (study 171/10), and all participants gave informed consent.

Data sharing: Study protocol and de-identified data are available from the corresponding author.

1 HIV, viral hepatitis and sexually transmissible infections in Australia. Annual Surveillance Report. Kirby Institute, 2012. www.kirby.unsw.edu.au/surveillance/2012-annualsurveillance-report-hiv-viral-hepatitis-stis.

2 Beyrer C, Baral SD, van Griensven F, Goodreau SM, Chariyalertsak S, Wirtz AL, et al. Global epidemiology of HIV infection in men who have sex with men. Lancet 2012;380:367-77.

3 CDC. HIV surveillance report 2010. Vol 22. Centers for Disease Control and Prevention, 2012.

4 Wilson DP. HIV treatment as prevention: natural experiments highlight limits of antiretroviral treatment as HIV prevention. PLoS Med 2012;9:e1001231.

5 Fox J, White PJ, Macdonald N, Weber J, McClure M, Fidler S, et al. Reductions in HIV transmission risk behaviour following diagnosis of primary HIV infection: a cohort of high-risk men who have sex with men. HIV Med 2009;10:432-8.

6 Gorbach PM, Weiss RE, Jeffries R, Javanbakht M, Drumright LN, Daar ES, et al. Behaviors of recently HIV-infected men who have sex with men in the year postdiagnosis: effects of drug use and partner types. J Acquir Immune Defic Syndr 2011;56:176-82.

7 Cohen MS, Chen YQ, McCauley M, Gamble T, Hosseinipour MC, Kumarasamy N, et al. Prevention of HIV-1 infection with early antiretroviral therapy. N Engl J Med 2011;365:493-505.

8 Marks G, Crepaz N, Janssen RS. Estimating sexual transmission of HIV from persons aware and unaware that they are infected with the virus in the USA. AIDS 2006:20:1447-50.

9 Charlebois ED, Das M, Porco TC, Havlir DV. The effect of expanded antiretroviral treatment strategies on the HIV epidemic among men who have sex with men in San Francisco. Clin Infect Dis 2011;52:1046-9. 


\section{What is already known on this topic}

Increased HIV testing in at risk populations is required to help stem further HIV transmission

Rates of testing in many countries remain low, with HIV remaining undiagnosed in a substantial proportion of infected people

\section{What this study adds}

In a randomised trial, men who have sex with men who were offered point of care testing for HIV did not have a significantly higher rate of HIV testing than those accessing conventional HIV testing over 18 months' follow-up

Post hoc analysis showed an initial increase in their rate of testing that was not sustained

10 Wilson DP, Hoare A, Regan DG, Law MG. Importance of promoting HIV testing for preventing secondary transmissions: modelling the Australian HIV epidemic among men who have sex with men. Sex Health 2009;6:19-33.

11 Birrell F, Staunton S, Debattista J, Roudenko N, Rutkin W, Davis C. Pilot of non-invasive (oral fluid) testing for HIV within a community setting. Sex Health 2010;7:11-6.

12 CDC. Prevalence and awareness of HIV infection among men who have sex with men21 cities, United States, 2008. MMWR Morb Mortal Wkly Rep 2010:59:1201-7.

13 Li X, Lu H, Raymond HF, Sun Y, Jia Y, He X, et al. Untested and undiagnosed: barriers to HIV testing among men who have sex with men, Beijing, China. Sex Transm Infect 2012;88:187-93.

14 Mallitt KA, Wilson DP, McDonald A, Wand H. HIV incidence trends vary between jurisdictions in Australia: an extended back-projection analysis of men who have sex with men. Sex Health 2012:9:138-43.

15 Moore DM, Kanters S, Michelow W, Gustafson R, Hogg RS, Kwag M, et al. Implications for HIV prevention programs from a serobehavioural survey of men who have sex with men in Vancouver, British Columbia: the ManCount study. Can J Public Health 2012;103:142-6.

16 Pedrana AE, Hellard ME, Wilson K, Guy R, Stoove M. High rates of undiagnosed HIV infections in a community sample of gay men in Melbourne, Australia. J Acquir Immune Defic Syndr 2012;59:94-9.

17 Saxton PJ, Dickson NP, Griffiths R, Hughes AJ, Rowden J. Actual and undiagnosed HIV prevalence in a community sample of men who have sex with men in Auckland, New Zealand. BMC Public Health 2012;12:92.

18 Burns F, Hart G. Increased HIV testing in men who have sex with men. BMJ 2012;344:e501.

19 STIGMA. Sexually transmitted infection guidelines for men who sex with men. STIs in Gay Men Action Group, 2010. http://stigma.net.au/resources/STIGMA_MSM_Testing_ Guidelines_2010.pdf.

20 Workowski KA, Berman S. Sexually transmitted diseases treatment guidelines. MMWR Recomm Rep 2010;59:1-110.

21 Chibo D, Kaye M, Birch C. HIV transmissions during seroconversion contribute significantly to new infections in men who have sex with men in Australia. AIDS Res Hum Retroviruses 2012;28:460-4.

22 Wawer MJ, Gray RH, Sewankambo NK, Serwadda D, Li X, Laeyendecker O, et al. Rates of HIV-1 transmission per coital act, by stage of HIV-1 infection, in Rakai, Uganda. $J$ Infect Dis 2005;191:1403-9.

23 Chow EP, Wilson DP, Zhang L. The rate of HIV testing is increasing among men who have sex with men in China. HIV Med 2012;13:255-63.

24 Kerr LR, Mota RS, Kendall C, Pinho Ade A, Mello MB, Guimaraes MD, et al. HIV among MSM in a large middle-income country. AIDS 2013;27:427-35

25 Zablotska I, Holt M, de Wit J, McKechnie M, Mao L, Prestage G. Gay men who are not getting tested for HIV. AIDS Behav 2012;16:1887-94.

26 Prestage G, Brown G, Keen P. Barriers to HIV testing among Australian gay men. Sex Health 2012:9:453-8.

27 Spielberg F, Branson BM, Goldbaum GM, Lockhart D, Kurth A, Celum CL, et al. Overcoming barriers to HIV testing: preferences for new strategies among clients of a needle exchange, a sexually transmitted disease clinic, and sex venues for men who have sex with men. J Acquir Immune Defic Syndr 2003;32:318-27.

28 Brown AE, Murphy G, Rinck G, Clewley JP, Hill C, Parry JV, et al. Implications for HIV testing policy derived from combining data on voluntary confidential testing with viral sequences and serological analyses. Sex Transm Infect 2009;85:4-9.

29 FDA approves first over-the-counter home-use rapid HIV test Food and Drug Administration. 2013. www.fda.gov/NewsEvents/Newsroom/PressAnnouncements/ ucm310542.htm

30 Chen MY, Bilardi JE, Lee D, Cummings R, Bush M, Fairley CK. Australian men who have sex with men prefer rapid oral HIV testing over conventional blood testing for HIV. Int $J$ STD AIDS 2010;21:428-30.

31 Anaya HD, Hoang T, Golden JF, Goetz MB, Gifford A, Bowman C, et al. Improving HIV screening and receipt of results by nurse-initiated streamlined counseling and rapid testing. $J$ Gen Intern Med 2008;23:800-7.

32 Donnell-Fink LA, Arbelaez C, Collins JE, Novais A, Case A, Pisculli ML, et al. Acceptability of fingerstick versus oral fluid rapid HIV testing: results from the universal screening for HIV infection in the emergency room (USHER Phase II) randomized controlled trial. $J$ Acquir Immune Defic Syndr 2012;61:588-92.

33 Metsch LR, Feaster DJ, Gooden L, Matheson T, Mandler RN, Haynes L, et al. Implementing rapid HIV testing with or without risk-reduction counseling in drug treatment centers: results of a randomized trial. Am J Public Health 2012;102:1160-7.

34 Myers JJ, Modica C, Dufour MS, Bernstein C, McNamara K. Routine rapid HIV screening in six community health centers serving populations at risk. $J$ Gen Intern Med 2009;24:1269-74.

35 Spielberg F, Branson BM, Goldbaum GM, Lockhart D, Kurth A, Rossini A, et al. Choosing HIV counseling and testing strategies for outreach settings: a randomized trial. $J$ Acquir Immune Defic Syndr 2005;38:348-55.

36 Apanovitch AM, McCarthy D, Salovey P. Using message framing to motivate HIV testing among low-income, ethnic minority women. Health Psychol 2003;22:60-7.

37 Sweat M, Morin S, Celentano D, Mulawa M, Singh B, Mbwambo J, et al. Community-based intervention to increase HIV testing and case detection in people aged 16-32 years in Tanzania, Zimbabwe, and Thailand (NIMH Project Accept, HPTN 043): a randomised study. Lancet Infect Dis 2011;11:525-32.

38 Wilson DP, Fairley CK, Sankar D, Williams H, Keen P, Read TR, et al. Replacement of conventional HIV testing with rapid testing: mathematical modelling to predict the impact on further HIV transmission between men. Sex Transm Infect 2011;87:588-93.

\section{Accepted: 09 August 2013}

\section{Cite this as: BMJ 2013;347:f5086}

This is an Open Access article distributed in accordance with the Creative Commons Attribution Non Commercial (CC BY-NC 3.0) license, which permits others to distribute, remix, adapt, build upon this work non-commercially, and license their derivative works on different terms, provided the original work is properly cited and the use is non-commercial. See: http://creativecommons.org/licenses/by-nc/3.0/. 


\section{Tables}

Table 1/ Characteristics of men who have sex with men at enrolment in study of effect of rapid point of care HIV tests on frequency of HIV testing in public health service in Australia. Figures are medians unless stated otherwise

Rapid HIV test $(n=200)$ Conventional HIV test $(n=200)$

Age (years)

30

Time since last HIV test (months) 29

No (\%) university educated

6

6

No of male sex partners in previous year $114(57)$ $99(50)$

No of male anal sex partners in previous year

10

8

No (\%) reporting any unprotected anal sex with casual partners in previous year

$89(46)$

$80(42)$ 
Table 2| Incidence rate of HIV testing over time among men who have sex with men according to allocation to rapid HIV test or conventional HIV test in public health service in Australia

\begin{tabular}{|c|c|c|c|c|c|c|c|c|}
\hline \multirow[b]{2}{*}{ Outcome } & \multicolumn{3}{|c|}{ Rapid HIV test } & \multicolumn{3}{|c|}{ Conventional HIV test } & \multirow{2}{*}{$\begin{array}{l}\text { Incidence rate ratio } \\
\qquad(95 \% \mathrm{Cl})\end{array}$} & \multirow[b]{2}{*}{$P$ value } \\
\hline & No of tests & Person years & Tests/year (95\% Cl) & No of tests & Person years & Tests/year $(95 \% \mathrm{Cl})$ & & \\
\hline $\begin{array}{l}\text { HIV tests over } 18 \\
\text { months }\end{array}$ & 469 & 288 & 1.63 (1.49 to 1.79$)$ & 396 & 278 & $1.42(1.29$ to 1.57$)$ & 1.15 (0.96 to 1.38$)$ & 0.12 \\
\hline $\begin{array}{l}\text { First HIV test after } \\
\text { enrolment test }\end{array}$ & 161 & 122 & $1.32(1.13$ to 1.54$)$ & 141 & 140 & $1.01(0.86$ to 1.19$)$ & $1.32(1.05$ to 1.65$)$ & 0.02 \\
\hline $\begin{array}{l}\text { Subsequent HIV tests } \\
\text { (excluding first tests) }\end{array}$ & 308 & 166 & 1.86 (1.66 to 2.07$)$ & 255 & 139 & 1.83 (1.62 to 2.07 ) & $1.01(0.86$ to 1.20$)$ & 0.90 \\
\hline
\end{tabular}




\section{Figures}

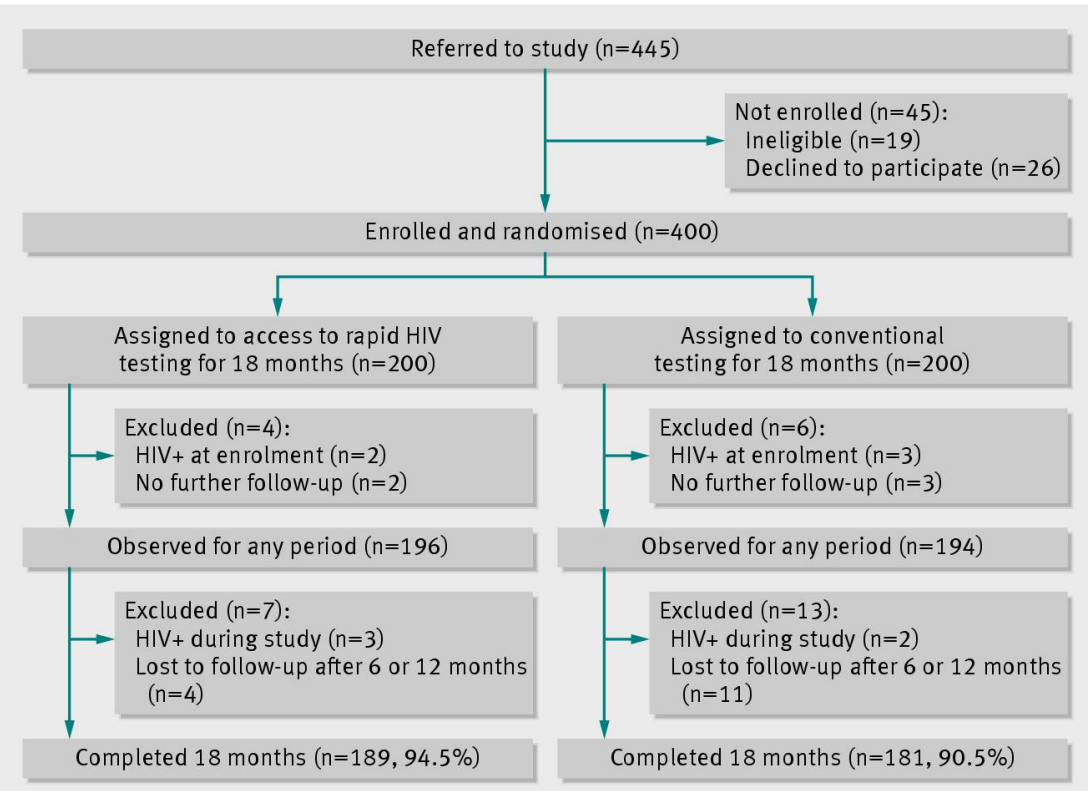

Fig 1 Screening, enrolment, randomisation, and follow-up of men who have sex with men in study of effect of provision of rapid HIV testing on frequency of tests

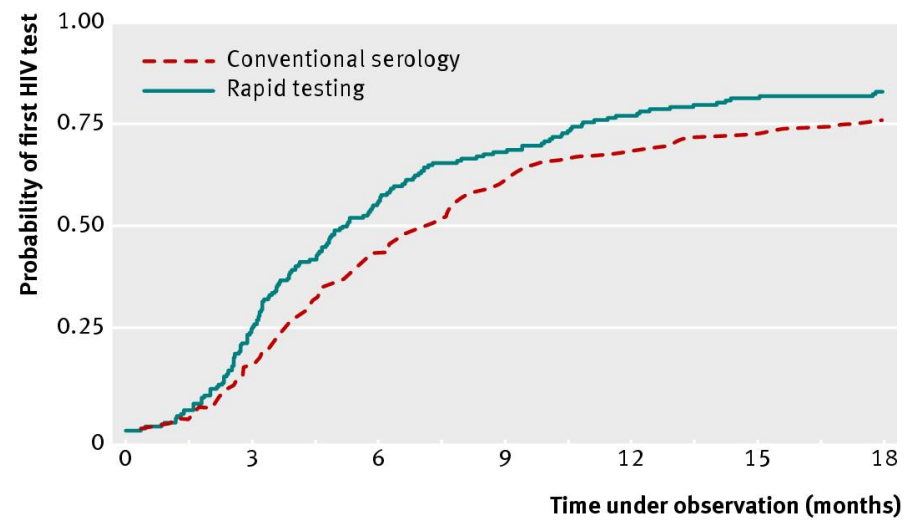

Fig 2 Kaplan-Meier estimates showing probability over time of first HIV test undertaken after baseline testing. Curve shows proportion of tests as they occur over 18 months 